\title{
NEURAL NETWORKS APPLIED TO PREVENTIVE CONTROL MEASURES FOR THE DYNAMIC SECURITY OF ISOLATED POWER SYSTEMS WITH RENEWABLES
}

\author{
J. N. Fidalgo, J. A. Peças Lopes (IEEE senior member), Vladimiro Miranda (IEEE member) \\ INESC - Instituto de Engenharia de Sistemas e Computadores \\ and DEEC/FEUP - Fac. de Engenharia da Universidade do Porto \\ P. República 93 - 4000 Porto, PORTUGAL \\ Fax: +351.2.2084172 email: jpl@obelix.fe.up.pt
}

\begin{abstract}
This paper presents an artificial neural network (ANN) based approach for the definition of preventive control strategies of autonomous power systems with a large renewable power penetration. For a given operating point, a fast dynamic security evaluation for a specified wind perturbation is performed using an ANN. If insecurity is detected, new alternative stable operating points are suggested, using a hybrid ANN-optimization approach that checks several feasible possibilities, resulting from changes in power produced by diesel and wind generators, and other combinations of diesel units in operation. Results obtained from computer simulations of the real power system of Lemnos (Greece) support the validity of the developed approach.
\end{abstract}

\section{INTRODUCTION}

Environmental concerns have been motivating increased interest in the use of renewable energy sources. In the European Union (EU) integration of renewable power production deserves a serious consideration. Whenever wind is available, and under the present market conditions, wind power is also an interesting economic option, specially in isolated power systems. In the EU there is a vast number of isolated systems, and the population living in medium and small islands is estimated to be over $3 \%$ of the population (over 10 million people).

In isolated systems global production costs of electricity can be reduced if wind power production is increased, particularly in cases where conventional production is provided by Diesel generators, characterized by high production costs that result not only from the high fuel price but also from the cost of fuel transportation. However, power systems that include wind power production are exposed to sudden wind speed changes. Corresponding production changes must be, fast and efficiently, compensated by the Diesel generators. Otherwise, large voltage and frequency variations, or even the collapse of the system, may result. Small isolated autonomous power systems are particularly sensitive to these disturbances due to natural wind variability. To avoid these problems, a very conservative policy of operation dispatch is usually adopted by local utilities, leading to increased spinning reserve (SR) requirements, to under exploitation of wind power production and to high production costs. In other cases, the same conservative security issues will restrain the construction of new wind farms.
Therefore, to increase wind power production, it is recommended to install advanced global control systems, connected with local SCADA. These systems will suggest the operating strategy for the upcoming hours by presenting to the system operators the start and stop schedule of the diesel machines and wind generators. These suggestions result from short time load and wind forecasts, and are based on minimization of global production costs, as well as on verification of a given degree of system dynamic security relative to some specified disturbances. Besides, dynamic security is monitored continuously and on-line during real operation.

The security tasks of the control system are: 1) assessment of security indices for given wind disturbances; and 2) definition of adequate preventive control actions, namely load redispatches and 3) determination of secure unit commitment (UC) alternatives.

This paper describes the security monitoring module, developed within the framework of the EU research program JOULE. A pilot control system that incorporates the approach here described is operating since January 1995 in the island of Lemnos, Greece. The description of the project and other modules, such as "wind and load prediction", "unit commitment and dispatch" may be found in [1].

In this project, ANNs are an essential piece. Not only do they perform consistently better than traditional statistical methods in the dynamic security pattern classification [2], but they also provide means for evaluating the degree of security. Moreover, they provide simple and effective mechanisms of computing the derivatives of a security index with respect to the control variables. Such ability allows the application of gradient based methods. In this paper, we show how these methods can be used to escape from dynamic unsafe states and achieve new secure ones.

\section{PREVIOUS WORK}

The application of ANNs for dynamic security assessment was previously used by Sobajic and Pao [3] for fast evaluation of critical clearing time. Kumar et al. [4] described in a general way the requirements needed by ANNs to evaluate dynamic security in large power systems. Djukanovic et al. [5] presented a new ANN approach for the determination of load shedding amount that assures transient stability. A good description of the application of ANN techniques in power systems can be found in [6].

Recently the authors of this paper presented a new ANN based method for transient stability assessment and preventive control [7, 8]. This approach evaluates the transient stability degree, on the basis of the emulation of the transient energy margin, and defines preventive control measures through a hybrid ANN-optimization method that solves an economic dispatch with transient stability constraints. This can practically be achieved through power exchanges among system generators. New stable operating points (OP) for one or several contingencies are found using a minimal incremental operation cost strategy [7, 8]. Results obtained using the CIGRE test system confirmed the feasibility of the developed approach. 
However in this approach, for some insecure operating points (load level, UC, etc.), it may happen that no stable state can be reached on the basis of only power exchanges among generators, i.e., these exchanges are not enough to bring the system into the stable domain.

In this paper, we describe how to extend the stable domain by considering new different UC alternatives. When an insecure OP is detected by the "security module", the searching algorithm looks for secure OPs not only on the basis of power exchanges among generators, but also exploiting new feasible UC alternatives, namely suggested or resulting from the interaction with the "UC and dispatch module". In the control system installed on Lemnos, an interactive dialogue may be established between the two modules, before a final decision is reached.

\section{THE BASICS}

It is not in the scope of the present paper to describe all the features of the approach, namely the selection of observation variables, ANN fundamentals or other intermediate steps. A detailed description can be found in $[7,8]$. However, for completeness, the method's basic features are summarized next.

The approach requires the following major steps:

1 - Selecting the ANN input variable set;

2 - Obtaining an ANN able to evaluate on-line the system dynamic security degree.

3 - Defining a penalty function to represent security constraints (or insecurity costs).

4 - Obtaining a new secure operating point using an algorithm (ALG0) that integrates both cost of production and security. At this stage a gradient based process is used to push the system towards a secure/economic state.

For Step 1, we required that the chosen set of variables would have the following properties:

i) adequately characterize the system state; this set should be small enough to avoid a large number of ANN parameters;

ii) be independent among them;

iii) include a subset of controllable variables so that it might be possible to exercise control actions in the system.

Moreover, these variables should be directly obtained from the SCADA data base to ease the monitoring and control procedures.

When properly trained, ANN output should be an accurate measure of a security index. The same ANN will also provide an efficient way of getting sensitivities of the output (security index) relative to the input variables, namely relative to the control variables. Then, a gradient based method is applied changing the control variables to increase security. Since control variables are power produced in each bus bar, then each iteration corresponds to a dispatch correction.

The algorithm ALG0 performs a gradient based approach where the direction of each step is affected by several factors: the production cost factor, the derivatives of each contingency index with respect to the control variables, the penalty function and the power generation limits constraints. These constraints are checked and dealt with during the iterative procedure, so that dispatch corrections always lead to feasible load flow solutions.

\section{FAST DYNAMIC SECURITY EVALUATION}

The global control system developed [1] requires that all information about units in service and dispatch corrections, obtained within a "security module", should be passed to the "unit commitment and dispatch module", where they are combined with other constraints such as minimum up and down times and starting costs. The "security module" behavior is the object of this paper.

\section{A. The Lemnos system}

The electric power system of Lemnos[1] comprises one diesel power station (DPS), two wind parks (WP), and two $(2 * 250 \mathrm{~kW})$ photovoltaic plants (PV). The DPS comprises five diesel units $(2 * 1.2$ MW and $3 * 2.7 \mathrm{MW}$; one of the $2.7 \mathrm{MW}$ units is only used when one of the others is in maintenance). The two WPs have the following nominal capacities:

$\mathrm{WP} 1: 8 * 55 \mathrm{~kW}+7 * 100 \mathrm{~kW}=1.14 \mathrm{MW}$;

WP2 : $12 * 225 \mathrm{~kW}=2.7 \mathrm{MW}$.

Each of the two PVs is rated at $250 \mathrm{~kW}$. The relative small size of PVs and the particularly fast response of their control systems lead to their influence on the system dynamic behavior being minor. Fig. 1 presents a simplified diagram, where $\mathrm{D}$ is a single diesel unit.

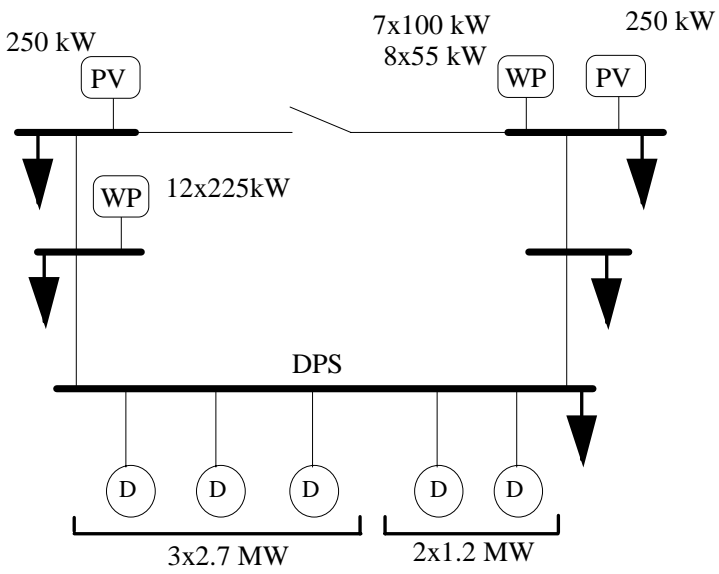

Fig. 1 - Single line diagram of Lemnos power system

\section{B. System security index}

If the frequency deviation caused by wind disturbances is greater than a certain threshold, protective devices are expected to disconnect wind farms or cause load shedding. Therefore the maximum absolute frequency deviation $(\Delta f)$ was chosen as the index that characterizes the system security. In this study, the system was considered secure if $\Delta \mathrm{f}<0.7 \mathrm{~Hz}$ (a safer security threshold) and insecure otherwise.

The evaluation of $\Delta \mathrm{f}$ index, for a given wind power disturbance, can be performed using a full dynamic security study. This evaluation needs the numerical solution of differential equations of the system with a complete representation of its components (asynchronous and synchronous generators with governor and voltage regulating devices). This demands a large computational effort, hopefully avoided by the use of ANNs in a real time environment.

\section{Feature selection and training conditions}

A data set has been generated by computer simulation by varying the total load (PL), the total wind power $(\mathrm{Pw})$ and the wind margin (WM). The WM is defined as the ratio of the diesel spining reserve to the total wind power. This data set comprised 1765 patterns. From these, 1059 were used for learning purposes, 353 for test and 353 for validation, using a random selection with uniform distribution. The stop training criterion adopted was based on the well known cross validation principle. Each operating point is characterized by a $\Delta \mathrm{f}$ value, obtained from a complete dynamic simulation performed for 
several seconds after a wind perturbation defined according to Fig. 2 . This has been chosen as the ANN output.

The set of system variables chosen as ANN inputs has been:

- Actual or forecasted load of the system PL;

- Actual or forecasted wind power production Pw;

- Nominal capacity of diesel units in operation Pdn.

These inputs are independent and controllable, allowing to control the effects in $\Delta \mathrm{f}$ (ANN output) from any change in them.

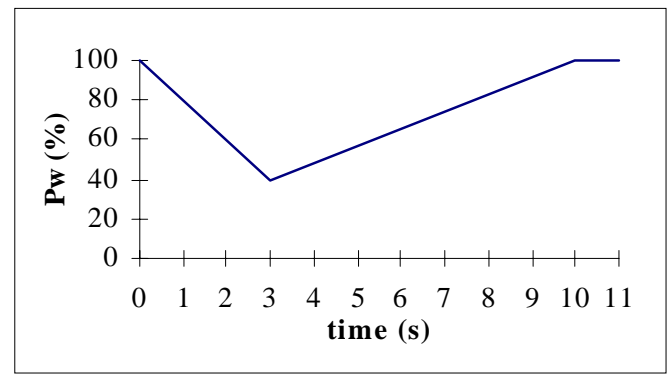

Fig. 2 - Wind power disturbance

Note that variable Pdn is directly related to the UC definition. There are 2 different types of diesel generators: G1 (1.2 MW) and G2 (2.7 MW). Table I represents the set of possible resulting combinations of machines in operation and corresponding Pdn value.

Table I

Pdn values for the several combinations of diesel units in operation

\begin{tabular}{ccc}
\hline nr. of G1 & nr. of G2 & Pdn (MW) \\
\hline 1 & 0 & 1.2 \\
0 & 1 & 2.7 \\
1 & 1 & 3.9 \\
2 & 0 & 2.4 \\
0 & 2 & 5.4 \\
2 & 1 & 5.1 \\
1 & 2 & 6.6 \\
2 & 2 & 7.8 \\
\hline
\end{tabular}

Since there is a biunique relation between the Pdn values and the set of diesel generators in operation, Pdn will be the "perfect" candidate as a control variable for the UC set-up.

The learning set was built representing a large spectrum of possible operating points, not only for the present conditions but also for some expected increase in load consumption or wind production in the near future. This will avoid the need of re-training the ANN continuously. Re-training could be necessary only if the load or generation diverge considerably from current operation scenarios. For major topologic changes such as adding a new line or power station then a new ANN must be built.

\section{ANN architecture and performance}

In this work, we have always used feedforward ANNs and the Adaptive Backpropagation (ABP) training algorithm [9]. The ABP is based in the traditional Backpropagation [10], but instead of a fixed and unique learning rate it uses an individual adaptive learning rate for each weight, which provides a much faster learning process.

The ANN has been built with the following strategy: 1- Building and training small ANN blocks, that establish relations among system variables with high discriminant power (for example, variables like wind margin can be easily obtained from PL, Pdn and Pw); 2- joining and fusing blocks and retraining the whole set as a single ANN. This approach lead to reduced training time and effort. The building blocks ANN1 to 3 and their dependencies are displayed in Fig. 3.

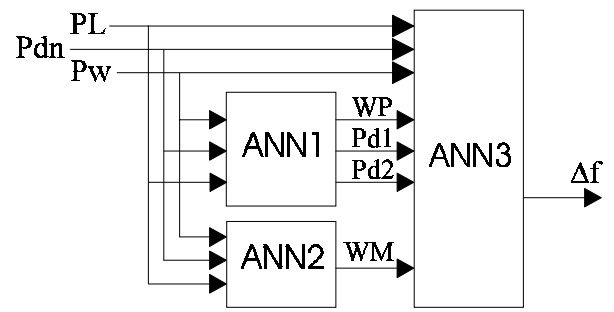

Fig. 3 - Building blocks of the ANN

After fusing the blocks into a single ANN and retraining, the architecture with the lowest mean square error in test set has been (3,8,4,4,1): 1st layer: 3 input units (PL, Pw and Pdn); 2nd layer: 8 units; 3rd layer: 4 units; 4 th layer: 4 units; last layer: 1 output unit $(\Delta \mathrm{f}$ value). The performance results in the test set were:

Table II

Performance evaluation results

\begin{tabular}{ll}
\hline mean absolute error & 0.00466 \\
mean relative error & 0.00825 \\
root mean square error & 0.00658 \\
\hline
\end{tabular}

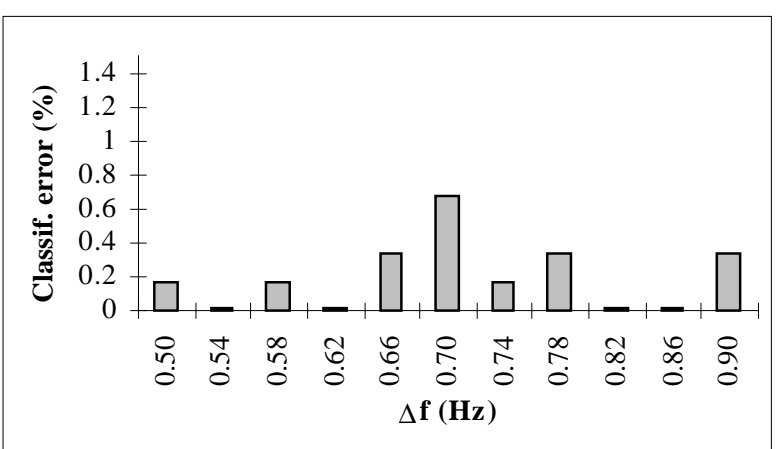

Fig. 4 - Classification error for several values of $\Delta \mathrm{f}$ threshold

The same ANN was used to perform security classification tasks and the error obtained in the test set for the established $\Delta \mathrm{f}$ limit $(0.7 \mathrm{~Hz})$ was $0.68 \%$ of misclassified patterns. In order to study the robustness of the ANN classification, we repeated the same classification task with several different values for the $\Delta \mathrm{f}$ limit. This concern was motivated by the all possible settings of the protective underfrequency relays. The result of this analysis is displayed in figure 4, and one can notice the independence of the ANN classification approach relative to the used separation threshold.

During the design stage, other Pattern Recognition Techniques, like the K Nearest Neighbors (KNN) method and Decision Trees (DT), obtained using an inference inductive procedure were also used for security classification purposes, as described in [2]. However, the results obtained with this ANN approach showed better performance, for the global classification error obtained, as described in table III.

Table III

Comparative performance results

\begin{tabular}{ll}
\hline KNN & $2.38 \%$ \\
DT & $5.59 \%$ \\
ANN & $0.68 \%$ \\
\hline
\end{tabular}


Although the ANN architecture can be considered a little complex with a relatively large number of hidden units, we have an amazingly small tool: only three input variables to represent a power system from a dynamic security point of view.

\section{E. System analysis}

An adequate algorithmic approach requires a good general understanding of system behavior. W are particularly interested in acquiring a better and more complete picture of how the variables PL, $\mathrm{Pdn}$ and $\mathrm{Pw}$ affect the $\Delta \mathrm{f}$ index, for the specified wind perturbation.

For that purpose, some graphs were developed, and in order to get a better definition for some curves, new operating points (PL, Pdn, Pw) were generated and added to existing ones.

The $\Delta \mathrm{f}$ value corresponding to each of the new operating points was evaluated by the trained ANN. As an example, we present figures 5 and 6 which display some aspects of the Lemnos dynamic system behavior.

These figures show that for $\mathrm{Pdn}=5.1 \mathrm{MW}$ and $\mathrm{Pdn}=5.4 \mathrm{MW}$, there are regions in these curves where an increase in wind power production favors the system dynamic behavior (by decreasing $\Delta f$ ), which constitutes an unexpected result, at first sight.

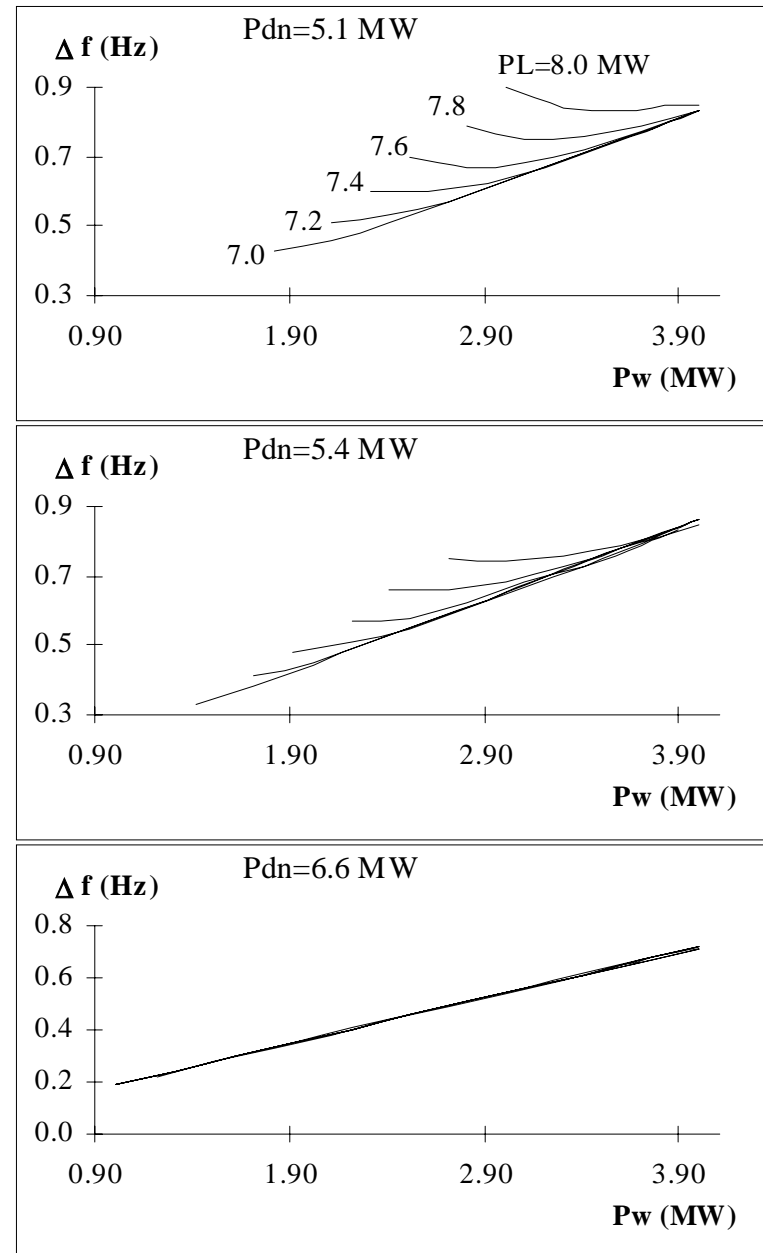

Fig. 5 - Family curves $\Delta \mathrm{f}=\mathrm{f}(\mathrm{Pw})$ for several values of $\mathrm{Pdn}$ (each graphic) and PL (each curve). Pdn, Pw and PL in MW
This particular behavior can be explained by analysis of the influence of Pw and SR in system dynamic security. Notice that, for fixed PL and Pdn, as higher Pw (higher wind penetration) becomes a greater $\Delta \mathrm{f}$ is expected, because the system becomes more vulnerable to wind changes. On the other hand, decreasing Pw implies a corresponding increase in the diesel power production (PD). If PD is close to Pdn, then SR becomes small and the system loses its compensating capabilities and becomes specially sensitive even to small changes in Pw. These facts make it evident that there are two compromising terms ( $\mathrm{Pw}$ and $\mathrm{SR}$ ) whose settings require careful tuning.

Without this knowledge, one might be tempted to implement an algorithm that simply reduces the wind power when a decreasing in $\Delta \mathrm{f}$ is desired; as we have just seen, that might result in an inappropriate approach.

One might also expect that the higher the total capacity of diesel machines in operation, the greater the wind margin would become, and, therefore, the expected frequency deviations would decrease. From observation of figure 6 one can see that this is not always true.

We can observe the crossing of some curves (notice curves for $\mathrm{Pdn}=5.1 \mathrm{MW}$ and $\mathrm{Pdn}=5.4 \mathrm{MW}$ ), which means that, in terms of dynamic security, the choice between these two values of Pdn must be made considering the values of PL and Pw.

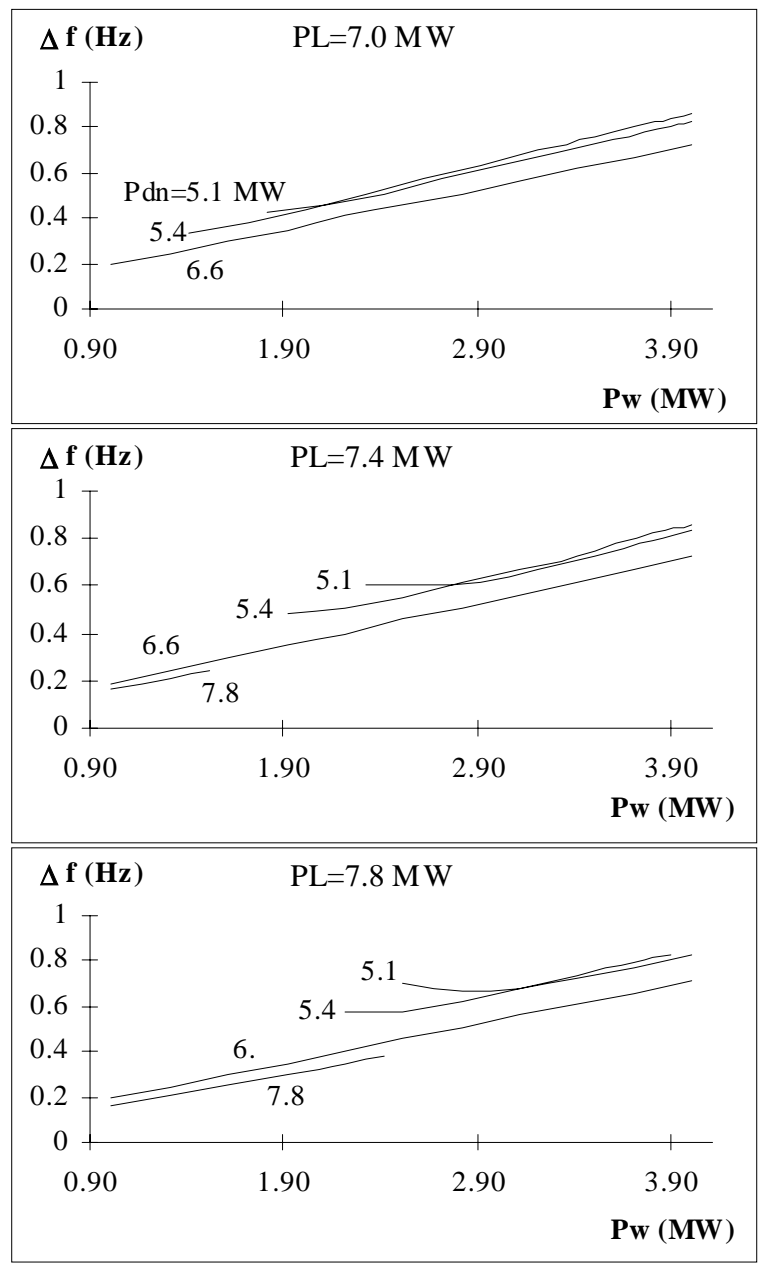

Fig. 6 - Curves $\Delta \mathrm{f}=\mathrm{f}(\mathrm{Pw})$ for several Pdn values with PL kept constant. Pdn, PL and Pw in MW 
If table I is analyzed, we may conclude that the two types of machines (G1 and G2) have a different dynamic security behavior. For example, for Pdn=5.1 MW, we have 2 G1 type machines and one G2 machine and for Pdn=5.4 MW, we have $2 \mathrm{G} 2$ machines. In spite of the higher value in the second case (providing a greater SR), the corresponding $\Delta \mathrm{f}$ value is not always smaller.

\section{F. Preventive Control}

This section describes an extension of the method previously reported to dynamic security assessment and control of autonomous power systems with diesel plus a large penetration of renewable energies. In this case the perturbation studied is the one described in figure 2. The focus will fall on study of the new possibilities of defining preventive control strategies by determining which Diesel units should be in operation. Again this is seen as a result of an interaction between the security and the UC modules of the control system.

Defining preventive control actions requires for each operating point and configuration:

a) accurate evaluation of $\Delta \mathrm{f}$, using the designed $\mathrm{ANN}$;

b) if $\Delta \mathrm{f}>0.7$ (unsafe), some changes in the control variables are required in order to decrease $\Delta \mathrm{f}$, moving the system towards its dynamic security domain; this job is performed by algorithm ALG1 and for all UC combinations suggested by the "unit commitment module".

ALG1 searches, for each detected insecure state, all the new set of combinations ( $\mathrm{Pdn}, \mathrm{Pw}$ ) corresponding to stable states. In pseudo code, it could conceptually be described by:

$$
\begin{aligned}
& \text { Input (initial state) } \\
& \text { if } \Delta \mathrm{f}>0.7 \text { then } \\
& \text { for all feasible values of } \mathrm{Pdn} \text { suggested by the } \mathrm{UC} \text { module } \\
& \quad \text { repeat } \\
& \qquad \Delta \mathrm{f}\left(\mathrm{P}_{\mathrm{W}}^{(\mathrm{n}+1)}\right)=\Delta \mathrm{f}\left(\mathrm{P}_{\mathrm{W}}^{(\mathrm{n})}\right)+\mathrm{h} \cdot \frac{\partial \Delta \mathrm{f}}{\partial \mathrm{P}_{\mathrm{W}}} ;
\end{aligned}
$$

until $\Delta \mathrm{f}<0.7$

where $\mathrm{n}$ is the iteration index and $\mathrm{h}$ is the gradient step. The derivative of $\Delta \mathrm{f}$ with respect to $\mathrm{Pw}$ is evaluated through the ANN, as described in $[7,8]$.

ALG1 allows changes in Pw and Pdn, for a specified load level PL. The new generated state is always a feasible state implicitly contained in the limits of the data set used to train the ANN.

The implementation of ALG1 was also based in the assumption that all diesel units were always available and:

$$
\begin{aligned}
& \text { 1. } \mathrm{P}_{\mathrm{dn}} \geq \sum_{\mathrm{i}} \mathrm{P}_{\mathrm{Di}} \\
& \text { 2. } \mathrm{P}_{\mathrm{Di}}^{\max } \geq \mathrm{P}_{\mathrm{Di}} \geq \mathrm{P}_{\mathrm{Di}}^{\min }
\end{aligned}
$$

where $\mathrm{P}_{\mathrm{Di}}, \mathrm{P}_{\mathrm{Di}}{ }^{\mathrm{max}}$ and $\mathrm{P}_{\mathrm{Di}}{ }^{\mathrm{min}}$ are respectively, the active power generated by diesel unit $\mathrm{i}$ and the maximum and minimum operation limits for each machine.

The table IV shows an example resulting from the application of ALG1. This particular example shows that we do not always need to change Pdn to obtain a new stable state. Instead, $\mathrm{Pw}$ is changed by disconnecting some wind generators. Notice also the paradoxical situation: in this case, when we have more diesel power available is when we may safely use the largest wind power with security.

Table IV

Initial state and new states provided by ALG1

\begin{tabular}{ccccc}
\hline & $\begin{array}{c}\mathrm{PL} \\
(\mathrm{MW})\end{array}$ & $\begin{array}{c}\mathrm{Pdn} \\
(\mathrm{MW})\end{array}$ & $\begin{array}{c}\mathrm{Pw} \\
(\mathrm{MW})\end{array}$ & $\begin{array}{c}\Delta \mathrm{f} \\
(\mathrm{Hz})\end{array}$ \\
\hline initial state & 7.6 & 5.1 & 4.00 & 0.83 \\
& & & & \\
\hline \multirow{2}{*}{ New } & 7.6 & 5.1 & 3.25 & 0.69 \\
states & 7.6 & 5.4 & 3.10 & 0.67 \\
& 7.6 & 6.6 & 3.80 & 0.69 \\
& 7.6 & 7.8 & 4.00 & 0.61 \\
\hline
\end{tabular}

The initial operation point resulting from an UC and dispatch without security concerns is labeled $\mathrm{Si}$. Starting from this point, the algorithm performs a search of new stable feasible states $\left(f_{k}, k=1 . .4\right)$. This search is guided, on one hand, by the derivative of $\Delta \mathrm{f}$ with respect to $\mathrm{Pw}$ and, on the other hand, by the knowledge acquired in the system analysis phase, related with the influence of Pdn on $\Delta \mathrm{f}$ value. For each value of Pdn considered, the algorithm changes $\mathrm{Pw}$ to decrease $\Delta \mathrm{f}$, and stops when $\Delta \mathrm{f}<0.7$. Operation costs have not been considered in this paper, (this task is addressed to the UC module, not described here). However, it is an easy task to evaluate costs for each new operating state and, from these results, a trade-off between security and operating cost can be obtained.

\section{G. Application of the method to the Madeira power system}

The presented methodology was also successfully applied to the power system of Madeira island. This network has a total installed capacity of 174 MVA, divided by one large Diesel power station (12 units of 4 different types), five hydro power plants and two large wind parks. Wind power penetration reaches $25 \%$ of total production during off-peak hours.

A learning set of about 3000 operating points, associated with the load diagram regions with higher wind penetration, was generated to obtain a good picture about the system dynamic behavior for a given set of wind disturbances. In this case a neural network with an architecture $(6,8,4,4,1)$ was used.

In spite of the larger dimension of the learning set no problems were detected in the ANN learning procedure. Also the performance of the obtained ANN was similar to the one obtained for Lemnos.

For larger systems the learning set dimension can be kept reduced if engineering judgment is previously used to identify particular operating conditions. For example operating strategies for peak load diagram periods or certain periods of the year (due to availability of energy resources) can be used. This approach aims at reducing the "limits of system operation" to be included in the learning set generation procedure [11]. As described in [11], the use of parallel processing methodologies can also be seen as a way to cope with the computational effort of the learning set generation phase.

\section{CONCLUSIONS}

ANNs provide easily built powerful tools for a large number of situations. In the example tested, ANNs were used not only to obtain fast and accurate assessment of the dynamic security index $\Delta \mathrm{f}$, but 
also as a decision aid tool to help in determining which Diesel machines should be in operation to assure dynamic security. ANNs had proved to be very useful when used as a regression tool for obtaining a complete graphic picture of system behavior.

The strategy adopted shows that simple but rich informative scenarios can be built to show the UC secure possible settings for a given system state. If each new proposed secure state is associated with its corresponding operating cost, then, we have an adequate picture of the trade-off between security level and costs.

The results obtained in this system are quite encouraging, supporting that:

1 - The present approach is a worthy application. It provides a user-friendly tool to solve the problem "secure UC settlement".

2 - The technique is easy to generalize. The method previously described in $[7,8]$ is here applied in another system, with a different kind of contingency and distinct system representation.

Therefore approaches such as the one described can lead to higher penetration of renewable energies and guarantee a secure operation of mixed Diesel and wind power systems. Also the increased use of renewables is very important from environmental and economic points of view.

\section{ACKNOWLEDGMENT}

The authors want to thank the research team of the project JOU2CT92-0053 of the European research program JOULE for their contributions in the previous phases, related with discussions and specifications of the problem addressed in this paper and particularly National Technical University of Athens and PPC (Public Power Corporation of Greece) for the data provided.

\section{REFERENCES}

[1] E. Nogaret et al., "Development and Implementation of an Advanced Control System for Medium Size Wind-Diesel Systems", Proc. European Wind Energy Conference, Thessaloniki, Greece, October 1994

[2] N. Hatziargyriou, S. Papathanassiou, J. A. Peças Lopes and V. Van Acker, "Pattern recognition versus decision tree methods a case study for fast dynamic secutiry assessment of autonomous power systems with a large penetration from renewables", presented to the ISAP'94 Conference, Montpellier, France, September 5-9, 1994

[3] D. J. Sobajic and Y. H. Pao, "Artificial neural-net based security assessment for electric power systems", IEEE Trans. on PWRS, Vol. 4, nr. 1, February 1989

[4] A. B. R. Kumar, A. Ipakchi, V. Brandwajn, M. El-Sharkawi and G. Cauley, "Neural network for dynamic security assessment of large-scale power systems: requirements overview", Proc. 1st Int. Forum on App. of Neural Networks to Power Systems, Seattle, USA, July 1991
[5] M. Djukanovic, D. J. Sobajic and Y. H. Pao, "Neural net based determination of generator shedding requirements in electric power systems", IEE Proc. C - Vol 139, nr. 5, Sep 1992

[6] Dagmar Niebur et al., "Artificial neural networks for power systems: A literature survey", Engineering Intelligent Systems, vol. 1, no. 3, Dec 1993

[7] J. N. Fidalgo, J. A. Peças Lopes, V. Miranda and L. B. Almeida, "Fast assessment of transient stability margins by a neural network approach", Proc. 11th PSCC, Avignon, France, Aug 1993

[8] V. Miranda, J.N. Fidalgo, J. A. Peças Lopes and L. B. Almeida, "Real Time Preventive control actions for transient stability enhancement with a hybrid neural network - optimization approach", IEEE Trans. on PWRS, Vol. 10, nº 2, May 1995

[9] F. M. Silva, L. B. Almeida, "Acceleration Techniques for the Backpropagation Algorithm", in "Neural Networks", L. B. Almeida and C. J. Wellekens (eds.], Springer-Verlag, 1990

[10] D. E. Rumelheart et al, "Learning Internal Representations by Error Propagation", in Parallel Distributed Processing: Explorations in the Microstructure of Cognition,. Rumelheart and MacCleland eds, MIT Press, Cambridge, MA, USA, 1986

[11] Y. Jacquemart, L. Wehenkel, T. Van Cutsem, P. Pruvot, "Statistical Approaches to Dynamic Security Assessment: The Data Base Generation Problem", To appear in the Proceedings of SIPOWER'95, IFAC Symposium on Control of Power Plants and Power Systems, Caucun, 6-8 December, 1995, Mexico

\section{BIOGRAPHIES}

J. N. Fidalgo was born in Oporto, Portugal, on August 30, 1961. He received his Licenciado degree from FEUP in 1984, in electrical engineering, when he joined INESC as a researcher. He is presently preparing his $\mathrm{Ph} . \mathrm{D}$. thesis. His research interests are neural networks and their application to power systems.

J. A. Peças Lopes was born in Sertã, Portugal, on May 23, 1958. He received his Licenciado and Ph.D. degrees from FEUP in 1981 and 88, in electrical engineering. In 1981 he joined FEUP and currently holds the position of Assistant Professor. He is also a senior researcher at INESC, in the IDEIA group. His research interests are power system security assessment and dispersed generation system analysis. He is a member of the CIGRE TF 38-06-06 on "Application of Artificial Neural Networks to Power Systems", and he is senior member of IEEE.

Vladimiro Miranda was born in Oporto, Portugal, on March 11, 1955. He received his Licenciado and Ph.D. degrees from the Faculty of Engineering (FEUP) of the University of Oporto in 1977 and 1982, all in electrical engineering. In 1981 he joined FEUP and currently holds the position of Associate Professor. In 1985 he joined also INESC - a Research Institute for Systems and Computers, and holds currently the position of Project Manager - Head of Information and Decision in Energy Systems Area (IDEIA). 